REVISTA

\title{
RECONFIGURAÇÃO INTELIGENTE DE REDES DE DISTRIBUIÇÃO DE ENERGIA ELÉTRICA CONSIDERANDO O INDICADOR FIC POR MEIO DO ALGORITMO BUSCA TABU
}

\author{
SMART RECONFIGURATION OF ELECTRICAL DISTRIBUTION \\ NETWORKS CONSIDERING THE FIC INDEX USING THE TABU SEARCH \\ ALGORITHM
}

\author{
Vitor Reuter Carréra Torres ${ }^{1}$ \\ Jussara Farias Fardin ${ }^{2}$ \\ Lucas Frizera Encarnação ${ }^{3}$ \\ Clainer Bravin Donadel ${ }^{4 *}$ \\ Rodrigo Fiorotti ${ }^{5}$ \\ Adan Lucio Pereira ${ }^{6}$ \\ Augusto César Rueda Medina ${ }^{7}$
}

\begin{abstract}
${ }^{1}$ Universidade Federal do Espírito Santo. E-mail: vitoreuter@hotmail.com.
${ }^{2}$ Universidade Federal do Espírito Santo. E-mail: jussara.fardin@ufes.br.

${ }^{3}$ Universidade Federal do Espírito Santo. E-mail: lucas@ele.ufes.br. ${ }^{4}$ Instituto Federal do Espírito Santo - campus Vitória. E-mail: cdonadel@ifes.edu.br. ${ }^{5}$ Instituto Federal do Espírito Santo - campus São Mateus. E-mail: rodrigo.fiorotti@ifes.edu.br. ${ }^{6}$ Universidade Federal do Espírito Santo. E-mail: adanlucio@gmail.com. ${ }^{7}$ Universidade Federal do Espírito Santo. E-mail: augusto.rueda@ufes.br.

*Autor para correspondência
\end{abstract}

Artigo submetido em 25/06/2019, aceito em 19/11/2019 e publicado em 23/12/2019.

Resumo: O processo de reconfiguração da rede elétrica visa atender a critérios técnico-econômicos como o número de consumidores atendidos, a redução das perdas, a manutenção dos níveis de tensão e carregamento do sistema em patamares adequados, a redução do número de manobras das chaves, dentre outros. Diversos métodos vêm sendo desenvolvidos a fim de determinar a configuração ótima para o sistema. Entretanto, diversos métodos propostos não consideram indicadores de continuidade do sistema como, por exemplo, a frequência de interrupção individual por unidade consumidora ou ponto de conexão (FIC), os quais podem acarretar multas pelo lado da distribuidora caso seus limites não sejam respeitados. Em outros casos, mesmo incluindo indicadores de continuidade tais como o FIC, os métodos propostos não conseguem evitar o desligamento repetido de unidades consumidores em caso de contingências sucessivas. Desta forma, é apresentada neste trabalho uma nova metodologia para reconfiguração das redes de distribuição de energia elétrica que atenda às restrições operacionais do sistema, incluindo o indicador de continuidade FIC, a fim de evitar que um mesmo conjunto de consumidores seja penalizado repetidamente em caso de desligamentos sucessivos. Para lidar com os múltiplos objetivos e restrições do problema de reconfiguração da rede elétrica de distribuição, a metodologia proposta foi baseada no Tabu Search Algorithm (Algoritmo Busca Tabu). O método proposto foi aplicado à rede IEEE 123 barras, rede padrão de testes estabelecida pelo IEEE (Institute of Electrical and Electronics Engineers), apresentando resultados coerentes com a proposta. 
Palavras-chave: Reconfiguração das redes de distribuição de energia elétrica; sistema de distribuição; Algoritmo Busca Tabu.

\begin{abstract}
The electrical distribution networks reconfiguration process aims to reach technical and economic criteria such as the number of served consumers, losses reduction, voltage and load adequate levels maintenance, switches changes reduction, among others. Several methods have been developed to determine the optimal system configuration. However, in several cases, the proposed methods do not consider system continuity indexes, such as the individual interruption frequency per consumer unit or connection point (FIC), which may result in penalties if their limits are not respected. In other cases, even including continuity indexes such as the FIC, the proposed methods cannot prevent repeated disconnection of consumer units in case of successive contingencies. In this way, this paper presents a new electrical distribution networks reconfiguration methodology that consider the system operating restrictions, including the FIC continuity index, avoiding that the same consumers group is repeatedly penalized in the event of successive contingencies. To address the multiple objectives and constraints of the distribution network reconfiguration problem, the proposed methodology was based on the Tabu Search Algorithm. The proposed method was applied to the IEEE 123 buses network, a standard test network established by the IEEE (Institute of Electrical and Electronics Engineers), presenting results consistent with the proposal.
\end{abstract}

Keywords: Electrical distribution networks reconfiguration; distribution system; Tabu Search Algorithm.

\section{INTRODUÇÃ̃O}

O consumo de energia elétrica per capita em qualquer país é um indicativo de seu crescimento e qualidade de vida de sua população (RAJARAM et al., 2015). À medida que o país se desenvolve, a necessidade por uma energia elétrica confiável e de qualidade vai ficando mais em evidência. Para que a energia elétrica seja considerada de boa qualidade, o sistema elétrico deve apresentar as seguintes características (BORGES et al., 2015): continuidade, ou seja, estar sempre disponível para uso; conformidade, atender aos padrões e normas técnicas especificados pelas agências reguladoras; flexibilidade, a fim de se adaptar às contínuas mudanças na estrutura topológica; manutenção, que é a capacidade da rede retornar ao modo de operação e fornecimento de energia o mais rápido possível, no caso de contingência na rede.

A rede de distribuição é o último estágio na entrega da energia elétrica. Ela é responsável por permitir o intercâmbio de energia entre o sistema de transmissão e o consumidor final. Atualmente, ela está ainda mais em evidência devido aos avanços das redes inteligentes (Smart Grids) e da geração distribuída (GD) que tornaram a rede ainda mais complexa e versátil, diante das possibilidades de melhorias que acrescentam ao sistema sendo, inclusive, um assunto que estará presente para estudo durante, pelo menos, a próxima década devido a sua relevância para o sistema elétrico (LÓPEZ et al., 2016).

Em geral, os sistemas de distribuição apresentam altos níveis de corrente e baixos níveis de tensão, o que acarreta perdas significativas de potência e níveis de tensão de baixa qualidade (NGUYEN et al., 2016). Paralelamente a tal situação, com o desenvolvimento das tecnologias de telecomunicação, automação e processamento de sinais digitais, chaves e dispositivos de proteção entre os alimentadores vêm evoluindo de forma a conseguir remotamente controlar e supervisionar o sistema, permitindo que este responda rapidamente às contingências (faltas) para um esquema de restauração automático (LÓPEZ et al., 2016). Diante de tal cenário, diversos métodos voltados para a reconfiguração de redes de distribuição vêm sendo desenvolvidos e aprimorados no intuito de mitigar tais perdas, melhorar os índices de qualidade da rede e responder de 
forma eficiente às contingências (ABDELAZIZ, 2017; BORGES et al., 2015; GHASEMI, 2018; LÓPEZ et al., 2016; MOHAMED IMRAN et al., 2014; NGUYEN et al., 2017; NGUYEN et al., 2016; RAJARAM et al., 2015; ZHAI et al., 2018).

A reconfiguração da rede de distribuição é o processo no qual o estado das chaves é alterado, visando modificar a topologia da rede de forma a respeitar as limitações impostas pelo operador (ALEMOHAMMAD et al., 2015; CHIDANANDAPPA et al., 2015; MOHAMED IMRAN et al., 2014). A alteração da topologia influencia no fluxo de potência da rede, o que impacta nas perdas do sistema, nos níveis de tensão, no carregamento da linha, dentre outros fatores. Os métodos lidam, então, com um problema de otimização não linear que pode conter vários objetivos distintos a serem tratados simultaneamente, de modo que o operador obtenha o desempenho ótimo para a rede (DUAN et al., 2015; ESMAEILIAN e FADAEINEDJAD, 2015). Estes processos de reconfiguração de redes de distribuição de energia elétrica podem ser aplicados de duas formas distintas: a primeira forma visa otimizar o funcionamento da rede elétrica em condições normais de operação, ou seja, quando toda a rede está operante e todos os consumidores estão sendo atendidos. A segunda forma visa buscar a restauração da rede elétrica de distribuição quando diante de contingências que não permitem $o$ atendimento a todas as unidades consumidoras. Neste caso, os métodos propostos de reconfiguração da rede elétrica, mesmo incluindo indicadores de continuidade tais como a frequência de interrupção individual por unidade consumidora ou ponto de conexão (FIC), não conseguem evitar o desligamento repetido de unidades consumidores em caso de contingências sucessivas. Assim, este artigo propõe uma nova metodologia de reconfiguração de redes de distribuição de energia elétrica que permita evitar a penalização do mesmo conjunto de consumidores, em situações de contingência que impossibilitem o atendimento a todos as unidades consumidoras.

O restante deste artigo está organizado da seguinte forma: na Seção 2 a metodologia proposta é apresentada. $\mathrm{Na}$ Seção 3 são apresentados os resultados obtidos pela aplicação do método proposto, bem como é apresentada uma discussão sobre sua eficácia. Por fim, na Seção 4, são apresentadas as conclusões deste trabalho.

\section{METODOLOGIA PROPOSTA}

Nesta seção é apresentada a metodologia proposta, incluindo os dados da rede utilizada, a descrição da função objetivo e do algoritmo de otimização utilizado.

\subsection{FUNÇÃO OBJETIVO}

O problema de reconfiguração de redes de distribuição de energia elétrica envolve as seguintes variáveis a serem otimizadas:

- Atendimento ao maior número de consumidores possível;

- Redução das perdas técnicas;

- Atender aos limites do indicador FIC.

E possui as seguintes restrições operacionais:

- Manter a topologia radial do sistema;

- Atender à capacidade de carregamento das linhas;

- Atender aos limites de tensão normatizados.

Desta forma, tem-se um problema multi-objetivo, onde os critérios apresentados anteriormente são conflitantes entre si. Entretanto, quando isso acontece, uma alternativa para a redução do problema é a utilização da técnica de ponderação de pesos, ou seja, vários objetivos são transformados em um único objetivo, criando uma abordagem ao problema monoobjetivo (COELLO et al., 2007). Para isso, é necessário definir uma função objetivo principal e uma função de avaliação que irá 
abordar os demais objetivos não descritos no objetivo principal. Assim, os objetivos "secundários" irão implicitamente influenciar na escolha de quais elementos irão compor a solução aplicada na função objetivo principal. O enfoque mono-objetivo é um ótimo correspondente às soluções extremas (mínimas ou máximas) da funçãoobjetivo, o que é desejado no problema em questão.

A escolha da função objetivo é de suma importância para a modelagem matemática do problema mono-objetivo e, para este trabalho, consiste em minimizar o custo total do ponto de vista da distribuidora de energia elétrica, garantindo que a reconfiguração da rede mantenha a estrutura radial, atenda aos limites legais e técnicos de tensão e corrente, além de permitir considerar a influência do indicador FIC. A função objetivo a ser minimizada é apresentada pela equação (1).

$$
\begin{aligned}
\text { cost }=\text { P.tec } & + \text { Carg.na } \\
& + \text { Lim. } . V+\text { Lim. I } \\
& + \text { FIC }
\end{aligned}
$$

objetivo são:

a) Perdas técnicas (P.tec): é a perda de potência ativa $(\mathrm{kW})$ da rede de distribuição, calculada pela equação (2) e pela equação (3).

$$
\text { P.tec }=\text { Perd } x \text { PreçokWc }
$$

$$
\begin{aligned}
\text { Perd }=V_{\text {slack }} I_{\text {slack }} f p_{\text {slack }} & \\
& -\sum_{i=1}^{n_{\text {cargas }}} V_{i} I_{i} f p_{i}
\end{aligned}
$$

Onde:

$V_{\text {slack }}$ Tensão no nó de início do alimentador;
$I_{\text {slack }}$ Corrente no início do alimentador;

$f p_{\text {slack }} \quad$ Fator de potência no início do alimentador;

$V_{i} \quad$ Tensão nos nós de carga;

$I_{i} \quad$ Corrente nos nós de carga;

$f p_{i} \quad$ Fator de potência nos nós de carga;

$n_{\text {cargas }}$ Quantidade de nós de carga existentes no alimentador;

PreçokWc Valoração das perdas de potência.

b) Custo das cargas não atendidas (Carg.na): é o quanto a distribuidora de energia elétrica deixa de faturar ao rejeitar determinadas cargas, dado pela equação (4). Esse fator estimula o algoritmo a atender, caso seja possível, ao máximo de clientes possíveis.

$$
\text { Carg.na }=\sum_{i=1}^{n a} V_{i} I_{i} x \text { PreçokWv }
$$

Onde:

na Cargas que não foram atendidas;

PreçokWv Valoração da carga não atendida.

c) Penalidade por infringir os limites de tensão (Lim.V): este indicador é ativado quando a distribuidora de energia elétrica abastece a unidade consumidora; entretanto, o fornecimento de energia elétrica ocorre fora dos limites estabelecidos por regulamentação. Foram adotados, neste trabalho, os limites estabelecidos pelo PRODIST Módulo 8 (BRASIL, 2018) para redes de média tensão, apresentados no Quadro 1. 
Quadro 1: Limites de tensão para redes de distribuição de energia elétrica de média tensão

\begin{tabular}{|c|c|}
\hline $\begin{array}{c}\text { Tensão de } \\
\text { Atendimento (TA) }\end{array}$ & $\begin{array}{c}\text { Faixa de Variação } \\
\text { de Tensão de } \\
\text { Leitura (TL) em } \\
\text { Relação à Tensão de } \\
\text { Referência (TR) }\end{array}$ \\
\hline Adequada & $\begin{array}{c}0,93 \mathrm{TR} \leq \mathrm{TL} \leq \\
1,05 \mathrm{TR}\end{array}$ \\
\hline Precária & $\begin{array}{c}0,90 \mathrm{TR} \leq \mathrm{TL} \leq \\
0,93 \mathrm{TR}\end{array}$ \\
\hline Crítica & $\begin{array}{c}\mathrm{TL}<0,90 \mathrm{TR} \text { ou TL }> \\
1,05 \mathrm{TR}\end{array}$ \\
\hline
\end{tabular}

Fonte: PRODIST - Módulo 8 (BRASIL, 2018).

Dessa forma, na função objetivo está sendo considerada uma penalidade quando o valor fica abaixo de $93 \%$ ou acima de $105 \%$ da tensão por unidade, ou seja, quando deixa de ser adequada e passar a ser precária ou crítica conforme apresentado na Figura 1.

Figura 1: Fluxograma de Determinação da Penalidade por Violação dos Limites de Tensão

Todos os nós nc da rede

energizada săo tais que

$0,93 \mathrm{pu} \leq \mathrm{Vi} \leq 1,05 \mathrm{pu}$ ?

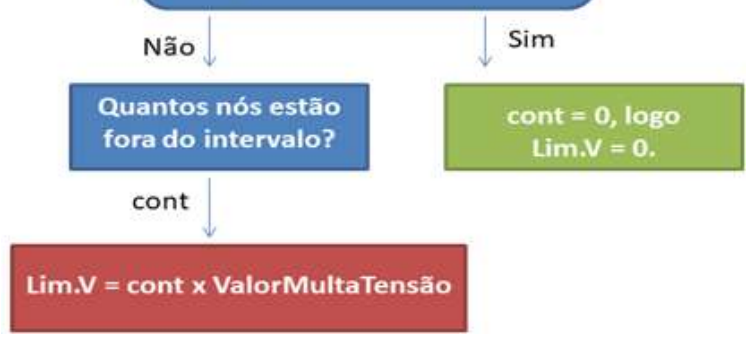

Fonte: Autores.

Onde:

$n c$

Número de nós com carga;

cont

Quantidade de cargas que estão sendo atendidas com tensão abaixo de 0,93TR ou acima de 1,05TR.

ValorMultaTensão Penalidade imposta à distribuidora de energia elétrica por desrespeitar os limites de tensão.

d) Penalidade por infringir o carregamento dos condutores ( $\mathrm{Lim} . \mathrm{I}$ ): esta é uma restrição de suma importância, uma vez que, caso seja descumprida, o condutor tende a se romper, o que poderá acarretar em prejuízos para a distribuidora de energia elétrica (por exemplo, possíveis acidentes, manutenção mais custosa e consumidores desenergizados) - equação (5).

\section{Lim. I$$
=\text { ValorPenalidadeCorrente }
$$

Onde:

Valor

Penalidade

Corrente

Valor de penalidade atribuído a função objetivo para os casos em que houve violação dos limites de corrente.

Vale ressaltar que não há um valor de penalidade previsto na legislação para este caso. Cabe a distribuidora de energia elétrica avaliar os riscos e estabelecer o valor de penalidade correspondente. Neste trabalho, foi estabelecido o valor de $10^{5}$ para tal penalidade, a fim de se evitar condutores operando em sobrecarga.

e) Penalidade por infringir o indicador de continuidade FIC (FIC): FIC representa a frequência de interrupção individual por unidade consumidora ou ponto de conexão, expressa em números de interrupção (BRASIL, 2018). Os limites para o indicador FIC são dependentes do tipo de unidade consumidora, do nível de tensão de atendimento e do valor dos indicadores de 
conjunto (DEC ou FEC). Neste trabalho foi adotado o valor dos indicadores de conjunto igual a 5 (valor típico para a Região Metropolitana da Grande Vitória), conforme é apresentado na Tabela 1, implicando em um limite para o indicador de continuidade FIC de 2,06 ocorrências/mês. O fluxograma de aplicação da penalidade por violação do indicador de continuidade FIC é apresentado na Figura 2.

Tabela 1: Limites de Continuidade por Unidade Consumidora - parte

\begin{tabular}{|c|c|c|c|c|c|c|c|}
\hline \multirow{4}{*}{$\begin{array}{l}\text { Faixa de variação dos } \\
\text { Limites Anuais de } \\
\text { Indicadores de } \\
\text { Continuidade dos } \\
\text { Conjuntos (DEC ou } \\
\text { FEC) }\end{array}$} & \multicolumn{7}{|c|}{$\begin{array}{c}\text { Limite de Continuidade por Unidade Consumidora ou Central } \\
\text { Geradora }\end{array}$} \\
\hline & \multicolumn{7}{|c|}{$\begin{array}{l}\text { Unidades Consumidoras ou Centrais Geradoras situadas em áreas } \\
\text { urbanas com Faixa de Tensão Contratada: } 1 \mathrm{kV}<\text { Tensão }<69 \mathrm{kV}\end{array}$} \\
\hline & \multicolumn{3}{|c|}{ DIC (horas) } & \multicolumn{3}{|c|}{ FIC (interrupções) } & \multirow{2}{*}{$\begin{array}{l}\begin{array}{c}\text { DMIC } \\
\text { (horas) }\end{array} \\
\text { Mensal }\end{array}$} \\
\hline & Anual & Trim. & Mensal & Anual & Trim. & Mensal & \\
\hline 1 & 11,25 & 5,62 & 2,81 & 6,48 & 3,24 & 1,62 & 2,36 \\
\hline 2 & 11,68 & 5,84 & 2,92 & 6,93 & 3,46 & 1,73 & 2,39 \\
\hline 3 & 12,12 & 6,06 & 3,03 & 7,37 & 3,68 & 1,84 & 2,41 \\
\hline 4 & 12,55 & 6,27 & 3,13 & 7,82 & 3,91 & 1,95 & 2,44 \\
\hline 5 & 12,99 & 6,49 & 3,24 & 8,27 & 4,13 & 2,06 & 2,46 \\
\hline ... & $\ldots$ & ... & $\cdots$ & $\ldots$ & $\ldots$ & $\cdots$ & $\ldots$ \\
\hline
\end{tabular}

Fonte: PRODIST - Módulo 8 (BRASIL, 2018).

Figura 2: Fluxograma de Aplicação da Penalidade por Violação do Indicador de Continuidade FIC

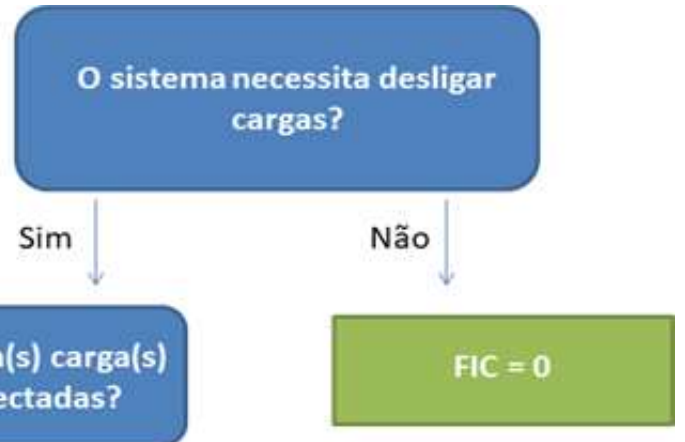

Grupo de W Cargas

Essas $W$ cargas já foram desligadas

alguma vez no período analisado?

Verificar na variável Interruption.

$\ln =0$

$\mathrm{FIC}=0$ $\ln \neq 0$

$F I C=$ ValorMultaFIC $\times \mathbf{W}$

Fonte: Autores. 
Onde In é uma abreviação da
variável contadora responsável por armazenar quantas vezes cada carga foi desligada no período mensal. Caso $o$ vetor Interruption( $i$ ) de determinado nó seja 1 (um) significa que ele já foi interrompido uma vez naquele mês, caso seja interrompido novamente irá implicar em multa. A variável ValorMultaFIC é dada pela equação (6) e é analisada para cada consumidor que foi desligado. Note que, caso o mesmo consumidor seja interrompido mais de uma vez dentro dos limites de FIC, quanto maior for o número de interrupções $(3,4 \ldots$ ao longo do mês), mais o valor da penalidade é acrescido. Isso irá garantir que o algoritmo busque não penalizar sempre a mesma unidade consumidora, mesmo estando dentro dos limites de FIC estabelecidos pela legislação.

$$
\begin{aligned}
& V A L O R_{F I C} \\
& =\left(\frac{F I C_{v}}{F I C_{p}}-1\right) D I C_{p}\left(\frac{C M}{730}\right) k e i
\end{aligned}
$$

Onde:

$F I C_{v}$ Frequência de interrupção por unidade consumidora ou por ponto de conexão, conforme cada caso, verificada no período considerado, expressa em número de interrupções;

FIC L $_{p}$ Limite de continuidade estabelecido no período considerado para o indicador de frequência de interrupção por unidade consumidora ou por ponto de conexão, expresso em número de interrupções e centésimo do número de interrupções;

$D I C_{p}$ Limite de continuidade estabelecido no período considerado para o indicador de duração de interrupção por unidade consumidora ou por ponto de conexão, expresso em horas e centésimos de hora;
CM Média aritmética dos encargos de uso do sistema de distribuição, considerando

também as demandas e energias reativas excedentes, correspondentes aos meses do período de apuração do indicador;

730 Número médio de horas no mês;

kei Coeficiente de majoração cujo valor deve ser fixado em: 15 (quinze), para unidade consumidora ou ponto de conexão atendidos em baixa tensão; 20 (vinte), para unidade consumidora ou ponto de conexão atendidos em média tensão; ou 27 (vinte e sete), para unidade consumidora ou ponto de conexão atendidos em alta tensão.

\subsection{ALGORITMO DE OTIMIZAÇÃO}

Os algoritmos Meta-Heurísticos têm tido grande aceitação dos autores da área de sistemas elétricos de potência (KAGAN et al., 2009). Eles são métodos que se baseiam nas técnicas de inteligência artificial e possuem como vantagem a facilidade para encontrar ótimos globais, independentemente do estado inicial, uma vez que realizam suas buscas em vizinhanças dentro do espaço de pesquisa, evitando paradas prematuras em ótimos locais. Dentre os diversos algoritmos meta-heurísticos encontrados na literatura, o Algoritmo Busca Tabu (em inglês, Tabu Search Algorithm) foi escolhido para o processo de otimização. Este algoritmo é um método eficiente para respostas discretas, o qual se encaixa no perfil do problema em questão, uma vez que a resposta desejada é uma matriz de estados das chaves composta por zeros (chaves abertas) e uns (chaves fechadas). Além disso, o Algoritmo Busca Tabu possui memória dinâmica, o qual aumenta a eficiência do processo de exploração e é extremamente útil para sair de máximos ou mínimos locais. $\mathrm{O}$ Algoritmo Busca Tabu é um método não populacional, isto é, avalia um candidato de cada vez e possui um sistema de memória dinâmica. O diferencial do sistema de 
memória da Algoritmo Busca Tabu é que ele não mantém apenas os valores das avaliações feitas até então, como também, o itinerário das últimas soluções visitadas (COELLO et al., 2007). Além disso, passa a ser possível identificar regiões promissoras do espaço de busca. O Algoritmo Busca Tabu é uma metaheurística para guiar heurísticas conhecidas a superar o problema da optimimalidade local. É baseado na premissa de que resoluções de problemas, para se qualificar como inteligentes, devem incorporar memória adaptativa e exploração dinâmica (SOLIMANPUR e ELMI, 2013).

\subsection{REDE DE TESTES UTILIZADA E CONDIÇÕES GERAIS DO FLUXO DE CARGA}

A rede de distribuição de energia elétrica utilizada para aplicação do método proposto é a rede IEEE 123 barras, mostrada na Figura 3.

Figura 3: Topologia da Rede de Distribuição IEEE 123 Barras

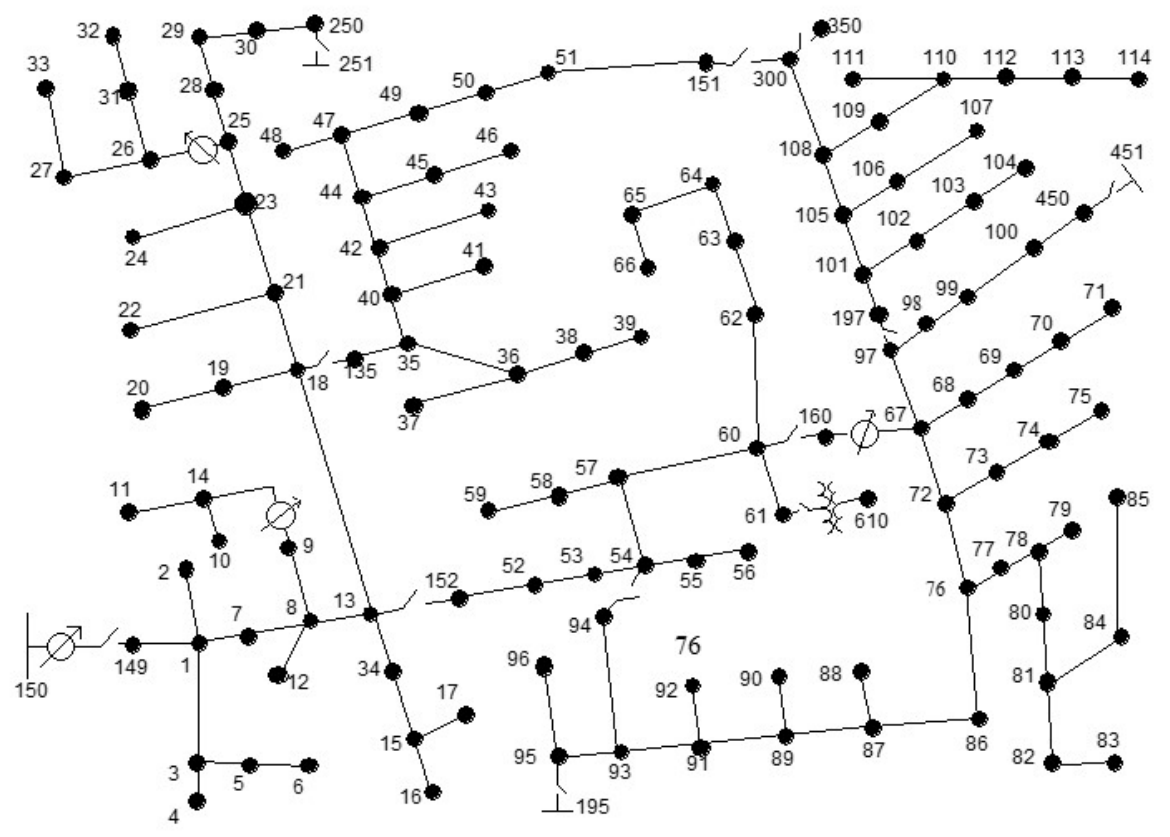

Fonte: Autores.

Entretanto, algumas modificações foram feitas de modo a adequar à situação desejada, conforme listado abaixo:

- Os transformadores foram simplificados, assim como o regulador de tensão localizado entre os nós 150 e 149.

- A rede original apresenta 4 alimentadores que abastecem todas as cargas do sistema. Entretanto, a efetividade da metodologia proposta somente pode ser constatada em caso de sobrecarga da rede, situação na qual parte da carga deve ser rejeitada. Dessa forma foram retirados três dos quatro alimentadores da rede $(195,451 \mathrm{e}$ 251), bem como os reguladores de tensão localizados no meio da rede, restando apenas $o$ alimentador localizado no nó 150 e o regulador de tensão localizado entre o mesmo e o nó 149 ;

- Cada aresta da rede (trecho) foi avaliada como uma posição candidata a receber uma, ou seja, no processo de reconfiguração foi concedida maior liberdade ao 
algoritmo, pois a rede original possui somente 6 chaves.

- O cabo que liga os nós 93 e 94 que é monofásico foi alterado para trifásico de modo que seja possível a utilização da chave para reconfiguração nos dois sentidos. Em sua forma original, somente o nó 54 poderia abastecer o nó 94 , já que o trecho entre os nós 93 e 94 é monofásico. Não é possível uma rede monofásica abastecer outra trifásica.

- Por fim, as cargas foram ajustadas, a fim de fazer com que o alimentador trabalhe em sobrecarga. Desse modo, este realizará rejeição de carga ao mesmo tempo que analisa o indicador FIC do sistema.

Para o cálculo do fluxo de carga, assume-se que a topologia da rede, a potência instalada (alimentador) e a potência nas cargas (ou correntes de carga) são conhecidas (KERSTING, 2002). Dessa forma, as condições operacionais da rede de distribuição de energia elétrica são conhecidas, uma vez que o fluxo de carga irá obter a tensão complexa em cada barra e os fluxos de potência (ativa e reativa) em todas as linhas ativas dessa rede e, portanto, as soluções geradas podem ser analisadas. $\mathrm{O}$ método utilizado foi o de varredura por soma de correntes, recomendado para cálculos em redes radiais (KAGAN et al., 2005). O algoritmo de fluxo de carga foi validade a partir da comparação entre os resultados obtidos para a rede IEEE 123 barras e os resultados-gabarito fornecidos pelo próprio IEEE junto às informações da rede, com diferenças inferiores à $0,1 \%$. As simulações foram realizadas no software MATLAB 2016b. O computador utilizado foi Intel $^{\circledR}$ Core тм i5 CPU M480 2,67GHz, 3,00GB RAM e foram utilizadas para cada simulação 500 iterações e uma lista tabu de tamanho 115.

\section{RESULTADOS E DISCUSSÃO}

\subsection{SIMULAÇÃO DO CASO BASE}

A definição do caso base visa estabelecer a condição operacional de referência, considerando que o alimentador consiga atender todos os nós de carga sem desrespeitar nenhuma das restrições impostas pelo algoritmo. Dessa forma, as cargas foram ajustadas para de modo que o alimentador trabalhe em seu limite operacional, porém ainda conseguindo atender a todos os nós consumidores, ou seja, sem rejeitar cargas. Os valores das cargas originais foram divididos pelo fator 1,442. Este valor foi calculado por meio da divisão entre o valor de corrente total da rede pela corrente máxima admissível do condutor existente no início do alimentador. Após o ajuste das cargas, a topologia da rede foi otimizada seguindo o método proposto neste trabalho. Naturalmente, neste caso base, não houve penalidades referentes aos 4 últimos termos da equação (1), pois não houve violação de limites operacionais nem rejeição de carga. A topologia resultante, referente ao caso base, está mostrado na Figura 4.

Alterações feitas pelo algoritmo na topologia da rede, em relação a topologia original:

- Fechamento da chave que conecta os nós 151 e 300 ;

- Abertura da aresta que conecta os nós 101 e 97;

- Fechamento da chave que conecta os nós 54 e 94;

- Abertura da aresta que conecta os nós 67 e 72 .

Caracterização da topologia do caso base:

- Apresentou perdas técnicas no valor de $44,83 \mathrm{~kW}$;

- Atendeu aos limites de carregamento dos condutores (Tabela 2). Note que a Fase A está operando próxima ao seu limite (453 A). 
- Manteve a topologia da rede como radial;
- As tensões foram mantidas na faixa adequada.

- Atendeu a todos os nós da rede;

Figura 4: Topologia da Rede referente ao Caso Base

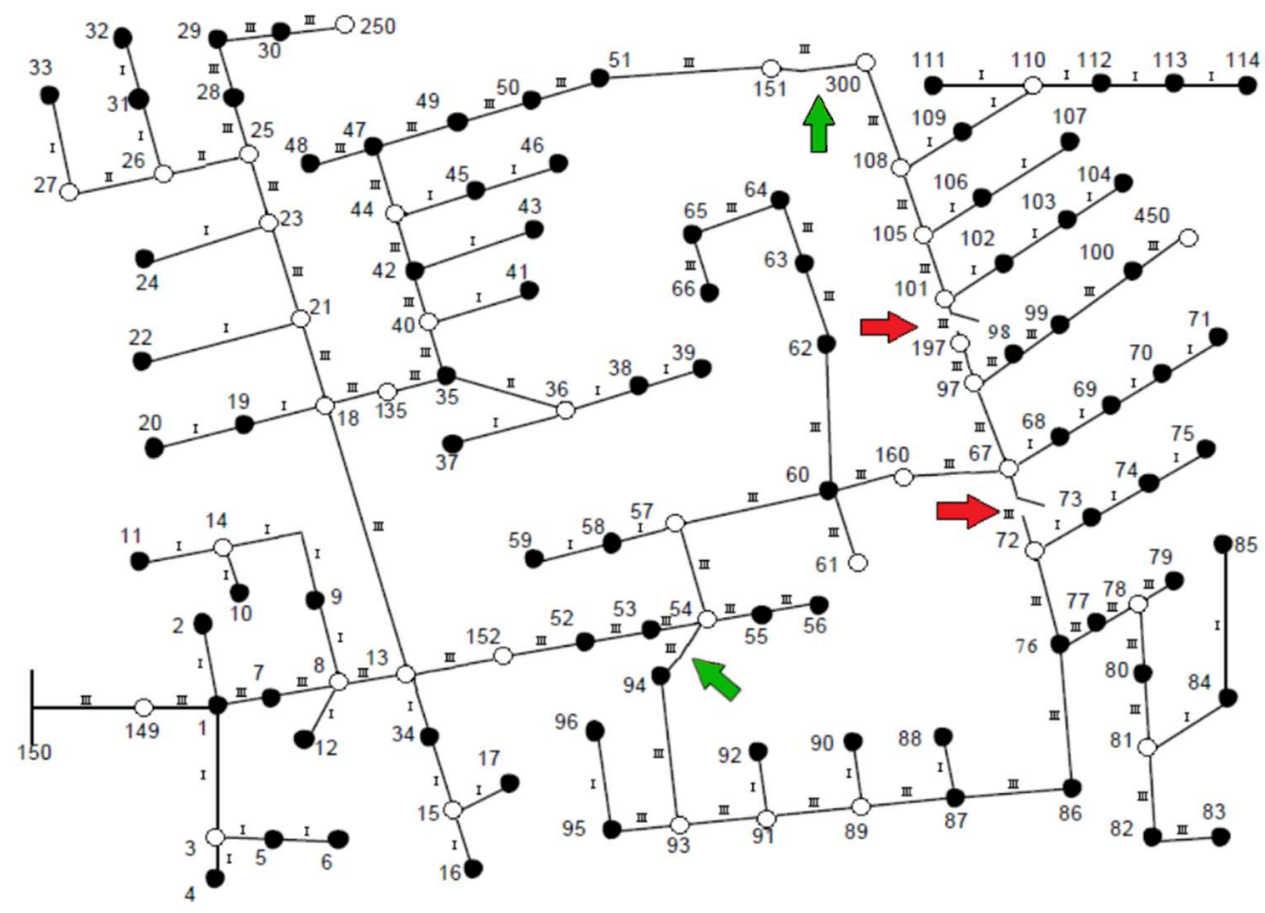

Fonte: Autores.

Tabela 2: Correntes por Fase no Início do Alimentador - Caso Base

\begin{tabular}{c|c|c}
\hline \multicolumn{3}{c}{ Corrente total (A) } \\
\hline Fase A & Fase B & Fase C \\
\hline 450,7 & 274,4 & 358,8 \\
\hline \multicolumn{3}{c}{ Fonte: Autores. }
\end{tabular}

\subsection{SIMULAÇÃO DOS CENÁRIOS DE SOBRECARGA}

2 cenários de sobrecarga foram simulados. As cargas foram ajustadas por dois fatores distintos, de modo a causar em ambas uma sobrecarga no sistema. Dessa maneira, espera-se que o algoritmo rejeite cargas para respeitas às restrições impostas na especificação do método proposto. Após a aplicação do ajuste de carga proposto referente ao cenário de sobrecarga \#1 e a otimização da topologia da rede, a topologia resultante é apresentada na Figura 5.
Caracterização da topologia referente ao cenário de sobrecarga \#1:

- Apresentou perdas técnicas no valor de 46,05 $\mathrm{kW}$;

- Atendeu aos limites de carregamento dos cabos (Tabela $3)$. Note que a Fase A está quase atingindo seu limite (453 A).

- Manteve a topologia da rede radial;

- Rejeitou as cargas dos nós 112, 113 e 114 para atender à restrição de carregamento. Vale ressaltar que o algoritmo optou por rejeitar cargas monofásicas da fase A (Tabela 4), de modo a preservar as cargas atendidas pelas outras fases, uma vez que a rede é desequilibrada com maior carregamento na fase A.

- As tensões foram mantidas na faixa adequada. 
Figura 5: Topologia da Rede referente ao Cenário de Sobrecarga \#1

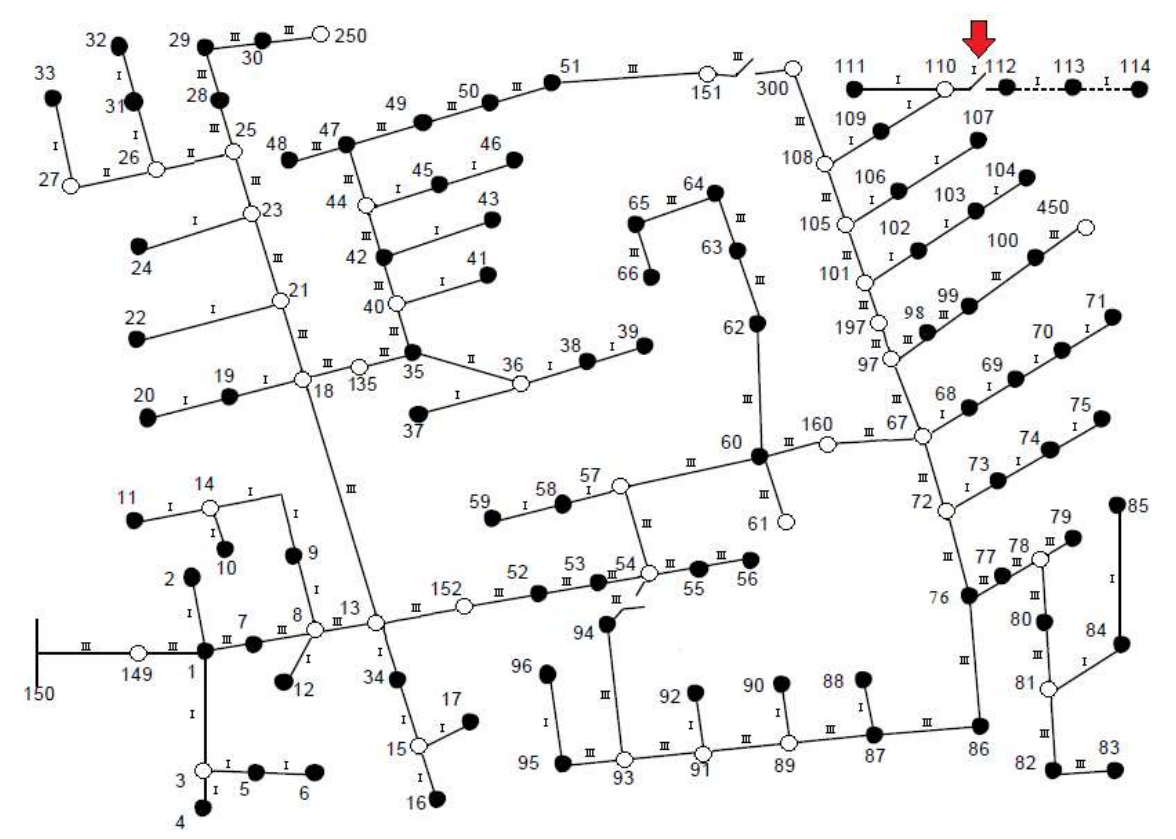

Fonte: Autores.

Tabela 3: Correntes por Fase no Início do Alimentador - Cenário de Sobrecarga \#1

\begin{tabular}{c|c|c}
\hline \multicolumn{3}{c}{ Corrente total (A) } \\
\hline Fase A & Fase B & Fase C \\
\hline 440,2 & 280,7 & 365,2 \\
\hline \multicolumn{3}{c}{ Fonte: Autores. }
\end{tabular}

Tabela 4: Carga não Atendida - Cenário de Sobrecarga \#1

\begin{tabular}{c|c|c}
\hline \multicolumn{3}{c}{ Carga não atendida (kW) } \\
\hline Fase A & Fase B & Fase C \\
\hline 56,34 & 0 & 0 \\
\hline \multicolumn{3}{c}{ Fonte: Autores. }
\end{tabular}

Após a aplicação do ajuste de carga proposto referente ao cenário de sobrecarga \#2 e a otimização da topologia da rede, a topologia resultante é apresentada na Figura 6.

Caracterização da topologia referente ao cenário de sobrecarga \#2:

- Apresentou perdas técnicas no valor de 45,58 $\mathrm{kW}$;
- Atendeu aos limites de carregamento dos condutores (Tabela 5). Note que a Fase A está quase atingindo seu limite (453 A).

- Manteve a topologia da rede radial;

- Rejeitou as cargas dos nós 113 e 114 para atender à restrição de carregamento (Tabela 6). Como a sobrecarga foi menor em relação ao cenário de sobrecarga $\# 1$, foi possível atender à carga conectada ao nó 112;

- As tensões foram mantidas na faixa adequada.

Tabela 5: Correntes por Fase no Início do Alimentador - Cenário de Sobrecarga \#2

\begin{tabular}{c|c|c}
\hline \multicolumn{3}{c}{ Corrente total (A) } \\
\hline Fase A & Fase B & Fase C \\
\hline 441,3 & 276,5 & 359,9 \\
\hline \multicolumn{3}{c}{ Fonte: Autores. }
\end{tabular}


Figura 6: Topologia da Rede referente ao Cenário de Sobrecarga \#2

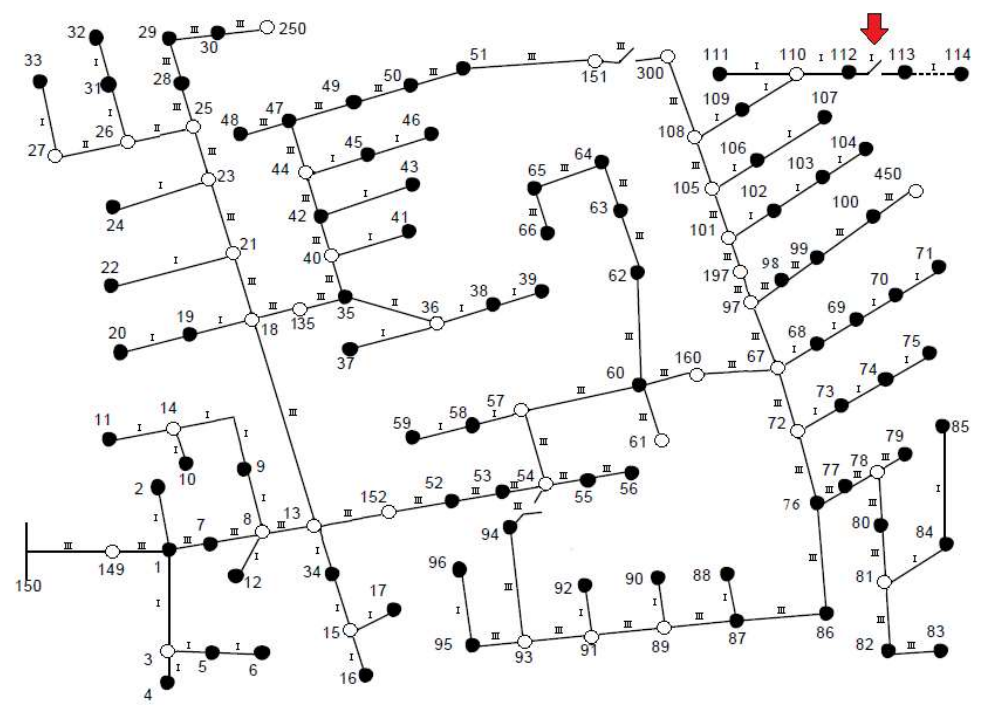

Fonte: Autores.

Tabela 6: Carga não Atendida - Cenário de Sobrecarga \#2

\begin{tabular}{c|c|c}
\hline \multicolumn{3}{c}{ Carga não atendida (kW) } \\
\hline Fase A & Fase B & Fase C \\
\hline 41,67 & 0 & 0 \\
\hline \multicolumn{3}{c}{ Fonte: Autores. }
\end{tabular}

Uma nova simulação foi realizada a partir do cenário de sobrecarga \#2, agora considerando o histórico de desligamentos nas cargas conectadas aos nós 113 e 114 . Entretanto, neste caso, poderá ocorrer penalização da função objetivo em relação a parcela FIC, uma vez que há histórico de desligamentos. Logo, o algoritmo deve avaliar se é vantajoso rejeitar cargas distintas às conectadas aos nós 113 e 144, ou rejeitar as mesmas cargas mesmo com a penalidade correspondente. Após esta nova simulação, a topologia resultante é apresentada na Figura 7.

Figura 7: Topologia da Rede referente ao Cenário de Sobrecarga \#2, considerando Histórico de Desligamentos

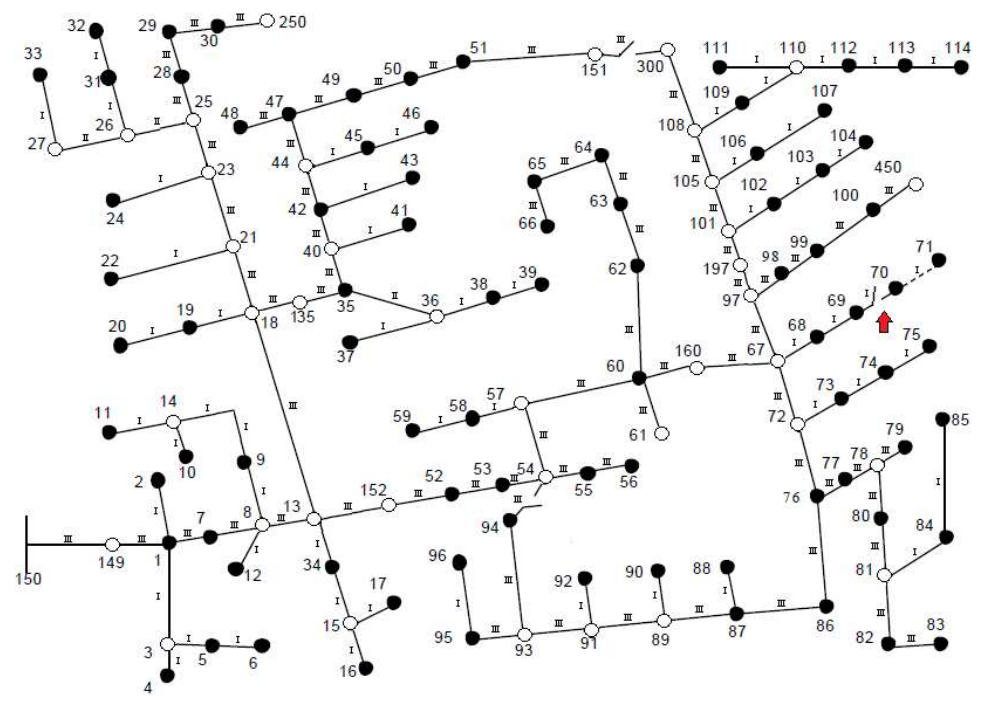

Fonte: Autores. 
Caracterização da topologia referente ao cenário de sobrecarga \#2, considerando o histórico de desligamentos:

- Apresentou perdas técnicas no valor de 45,88 kW;

- Atendeu aos limites de carregamento dos cabos (Tabela 7). Note que a Fase A está quase atingindo seu limite (453 A):

- Manteve a topologia da rede radial;

- Rejeitou, dessa vez, as cargas dos nós 70 e 71 para atender à restrição de carregamento e não ser penalizado devido à violação da parcela FIC da função objetivo, por desligar novamente as cargas conectadas aos nós 113 e 114 (Tabela 8);

- As tensões foram mantidas na faixa adequada.

Tabela 7: Correntes por Fase no Início do Alimentador - Cenário de Sobrecarga \#2 considerando Histórico de Desligamentos

\begin{tabular}{c|c|c}
\hline \multicolumn{3}{c}{ Corrente total (A) } \\
\hline Fase A & Fase B & Fase C \\
\hline 441,5 & 276,5 & 359,9 \\
\hline
\end{tabular}

Fonte: Autores.

Tabela 8: Carga não Atendida - Cenário de Sobrecarga \#2 considerando Histórico de Desligamentos

\begin{tabular}{c|c|c}
\hline \multicolumn{3}{c}{ Carga não atendida (kW) } \\
\hline Fase A & Fase B & Fase C \\
\hline 41,67 & 0 & 0 \\
\hline \multicolumn{3}{c}{ Fonte: Autores. }
\end{tabular}

Vale ressaltar que a soma das cargas monofásicas 113 e 114 são equivalentes às cargas 70 e 71, sendo os nós localizados no final das linhas e possuindo a mesma topologia de impedância. Entretanto, as cargas conectadas aos nós 113 e 114 foram desconectadas inicialmente (na situação ainda sem histórico de desligamentos) por estarem mais distantes do início do alimentador, implicando em perdas técnicas maiores ao longo do percurso.

\section{CONCLUSÕES}

Este artigo propôs uma nova metodologia de reconfiguração de redes de distribuição de energia elétrica que permita evitar a penalização do mesmo conjunto de consumidores, em situações de contingência que impossibilitem o atendimento a todos as unidades consumidoras. Este trabalho permitiu alcançar resultados promissores quanto ao processo de reconfiguração de redes de distribuição de energia elétrica, tema presente no cotidiano das distribuidoras e que interessa tanto o lado do consumidor, quanto o lado da própria distribuidora. Perdas técnicas, atendimento ao máximo de consumidores possível e limites de carregamento e tensão são elementos presentes com certa regularidade na publicação dos autores da área. Este trabalho, por sua vez, além de trabalhar com todos estes elementos, ainda adicionou métrica relacionada ao indicador de continuidade $F I C$, de forma diferenciada ao encontrado na literatura.

O método proposto foi capaz de analisar as restrições impostas e propor uma topologia radial eficiente para a situação. Desta forma, o algoritmo proposto não penalizou o mesmo grupo de clientes ainda que fosse mantido o valor de sobrecarga no sistema. Os resultados mostraram que o desligamento do mesmo bloco de cargas quando a rede está sobrecarregada pode ser evitado. Associado a isso, os resultados também mostraram que o Algoritmo Busca Tabu, utilizado no processo de otimização, apresentou bons resultados para respostas discretas e foi essencial para a otimização da rede. 


\section{AGRADECIMENTOS}

Os autores agradecem ao apoio institucional do Instituto Federal do Espírito Santo (Ifes) e da Universidade Federal do Espírito Santo (Ufes).

\section{REFERÊNCIAS}

ABDELAZIZ, M. Distribution network reconfiguration using a genetic algorithm with varying population size. Electric Power Systems Research, v. 142, p. 9-11, 2017/01/01/ 2017. ISSN 0378-7796.

Disponível em: <

http://www.sciencedirect.com/science/articl e/pii/S0378779616303273 >.

ALEMOHAMMAD, S. H.; MASHHOUR, E.; SANIEI, M. A market-based method for reconfiguration of distribution network.

Electric Power Systems Research, v. 125, p. 15-22, 2015/08/01/2015. ISSN 0378-

7796. Disponível em: <

http://www.sciencedirect.com/science/articl e/pii/S0378779615000814 >.

BORGES, G. P. et al. Use of genetic algorithm for evaluation and control of technical problems due load shedding in power systems. 2015 IEEE PES

Innovative Smart Grid Technologies Latin America (ISGT LATAM), 2015, 57 Oct. 2015. p.717-722.

BRASIL. Procedimentos de Distribuição de Energia Elétrica no Sistema Elétrico Nacional (PRODIST) - Módulo 8 Qualidade da Energia Elétrica de 01 de Janeiro de 2018. Brasília 2018.

CHIDANANDAPPA, R.;

ANANTHAPADMANABHA, T.; H.C, R.

Genetic Algorithm Based Network

Reconfiguration in Distribution Systems with Multiple DGs for Time Varying Loads. Procedia Technology, v. 21, p. 460-467, 2015/01/01/ 2015. ISSN 2212-0173.

Disponível em: <

http://www.sciencedirect.com/science/articl e/pii/S2212017315002510>.

COELLO, C. C.; LAMONT, G. B.; VELDHUIZEN, D. A. V. Evolutionary Algorithms for Solving Multi-Objective Problems. Springer US, 2007.
DUAN, D.-L. et al. Reconfiguration of distribution network for loss reduction and reliability improvement based on an enhanced genetic algorithm. International Journal of Electrical Power \& Energy Systems, v. 64, p. 88-95, 2015/01/01/ 2015. ISSN 0142-0615. Disponível em: < http://www.sciencedirect.com/science/articl e/pii/S0142061514004682 >.

ESMAEILIAN, H. R.; FADAEINEDJAD, R. Distribution system efficiency improvement using network reconfiguration and capacitor allocation. International Journal of Electrical Power \& Energy Systems, v. 64, p. 457-468, 2015/01/01/ 2015. ISSN 0142-0615. Disponível em: < http://www.sciencedirect.com/science/articl e/pii/S0142061514004098 $>$.

GHASEMI, S. Balanced and unbalanced distribution networks reconfiguration considering reliability indices. Ain Shams

Engineering Journal, v. 9, n. 4, p. 15671579, 2018/12/01/ 2018. ISSN 2090-4479.

Disponível em: <

http://www.sciencedirect.com/science/articl e/pii/S2090447916301617 >.

KAGAN, N.; OLIVEIRA, C. C. B. D.; ROBBA, E. J. Introdução aos Sistemas de Distribuição de Energia Elétrica. $1^{\mathrm{a}}$ Edição. São Paulo: Blucher, 2005. KAGAN, N.; SCHMIDT, H. P.; OLIVEIRA, C. C. B. D. Métodos de Otimização Aplicados a Sistemas Elétricos de Potência. $9^{a}$ Edição. São Paulo: Blucher, 2009.

KERSTING, W. H. Distribution System Modeling and Analysis. CRC Press, 2002.

LÓPEZ, J. C.; LAVORATO, M.; RIDER, M. J. Optimal reconfiguration of electrical distribution systems considering reliability indices improvement. International Journal of Electrical Power \& Energy Systems, v. 78, p. 837-845, 2016/06/01/ 2016. ISSN 0142-0615. Disponível em: < http://www.sciencedirect.com/science/articl e/pii/S014206151500575X $>$. MOHAMED IMRAN, A.; KOWSALYA, M.; KOTHARI, D. P. A novel integration technique for optimal network 
reconfiguration and distributed generation placement in power distribution networks. International Journal of Electrical Power \& Energy Systems, v. 63, p. 461-472, 2014/12/01/ 2014. ISSN 0142-0615.

Disponível em: < http://www.sciencedirect.com/science/articl e/pii/S0142061514003585 >.

NGUYEN, T. T. et al. Multi-objective electric distribution network reconfiguration solution using runner-root algorithm.

Applied Soft Computing, v. 52, p. 93-108, 2017/03/01/ 2017. ISSN 1568-4946.

Disponível em: <

http://www.sciencedirect.com/science/articl e/pii/S1568494616306366 >.

NGUYEN, T. T.; TRUONG, A. V.; PHUNG, T. A. A novel method based on adaptive cuckoo search for optimal network reconfiguration and distributed generation allocation in distribution network.

International Journal of Electrical Power \& Energy Systems, v. 78, p. 801-815, 2016. ISSN 0142-0615. Disponível em: < http://www.sciencedirect.com/science/articl e/pii/S0142061515005827 >.
RAJARAM, R.; SATHISH KUMAR, K.; RAJASEKAR, N. Power system reconfiguration in a radial distribution network for reducing losses and to improve voltage profile using modified plant growth simulation algorithm with Distributed Generation (DG). Energy Reports, v. 1, p. 116-122, 2015/11/01/ 2015. ISSN 23524847. Disponível em: < http://www.sciencedirect.com/science/articl e/pii/S2352484715000165 >.

SOLIMANPUR, M.; ELMI, A. A tabu search approach for cell scheduling problem with makespan criterion. International Journal of Production Economics, v. 141, n. 2 , p. 639-645, 2013/02/01/ 2013. ISSN 0925-5273. Disponível em: <

http://www.sciencedirect.com/science/articl e/pii/S0925527312004203 >.

ZHAI, H. F. et al. Dynamic reconfiguration of three-phase unbalanced distribution networks. International Journal of Electrical Power \& Energy Systems, v. 99, p. 1-10, 2018/07/01/ 2018. ISSN 01420615. Disponível em: < http://www.sciencedirect.com/science/articl e/pii/S0142061517321555 >. 